\title{
Ongoing tuberculosis transmission to children in Greenland
}

\author{
B. Soborg*, A. Koch*, V.Ø. Thomsen\#, K. Ladefoged", M. Andersson*, J. Wohlfahrt*, \\ M. Melbye* and A.B. Andersen ${ }^{+}$
}

ABSTRACT: Inuit in the Arctic are experiencing an increase in tuberculosis cases, reaching levels in Greenland comparable to high-incidence countries. This prompted us to study the level of tuberculosis transmission to Greenlandic children. Specifically, we estimated the current prevalence of Mycobacterium tuberculosis infection (MTI) and the underlying annual risk of MTI.

2,231 Greenlandic school children aged $5-17$ yrs $(\sim 25 \%$ of the Greenlandic population in the relevant age group) were tested for MTI using the tuberculin skin test and the QuantiFERON ${ }_{\mathbb{B}}$-TB Gold in-tube test. Subjects with dual-positive results were considered infected and subjects with dual-negative results uninfected. The children with discordant test results were classified as probably having MTI and analysed separately.

$8.1 \%$ of the children had dual-positive test results. The annual risk of MTI was estimated as $0.80 \%(95 \% \mathrm{Cl} 0.67-0.92 \%)$ giving a cumulative risk at the 18 th birthday of $13.4 \%$. The annual risk of MTI varied substantially by ethnicity $(0.87 \%$ in Inuit children, $0.02 \%$ in non-Inuit children; $p<0.001)$ and by location $(0.13 \%$ on the west coast, $1.68 \%$ on the south coast; $p<0.001)$.

M. tuberculosis transmission occurs at a very high level in Inuit children with pronounced geographic differences emphasising the need for immediate public health interventions.

KEYWORDS: Children, epidemiological study, tuberculosis, tuberculosis control

$\mathrm{n}$ the 1950s, the Inuit population in Greenland,

Alaska and Canada presented the highest

tuberculosis (TB) incidence rates ever observed [1]. In 1952, Greenland reported a TB incidence rate of 2,200 cases per 100,000 inhabitants [2]. A remarkable public health effort led to a $17 \%$ annual decline in TB incidence and by the mid-1980s, the levels were comparable with western countries [2] Consequently, the Greenlandic authorities scaled down preventive activities, including abandoning routine neonatal Bacille Calmette-Guérin (BCG) vaccination in 1991. However, after a short hiatus, the incidence rate reversed and in the 1990s, the TB incidence rate more than doubled [3]. Among TB cases were young children with TB meningitis and fatal outcome. In 1997, the National Board of Health in Greenland reintroduced neonatal BCG vaccination and in 2000 revived efforts aiming at disease control. Even so, in 2001, the TB incidence rate reached 185 cases per 100,000 , with $30 \%$ of cases diagnosed among children $<15$ yrs old [4] However, it is unknown whether the apparent increase in TB cases reflects disclosure of undiagnosed, prevalent cases infected decades earlier, or whether it reflects ongoing transmission.

The incidence of Mycobacterium tuberculosis infection (MTI) within a community can be approximated by the annual risk of $M$. tuberculosis infection (ARTI) [5]. ARTI is an indicator of ongoing TB transmission in the community and a proxy indicator of the effectiveness of preventive efforts [6]. ARTI is usually estimated on the basis of childhood tuberculin skin test (TST) surveys [79]. However, a positive TST result may be of limited predictive value because of cross-reactivity to the BCG vaccine and potentially to nontuberculous mycobacteria [10, 11]. The newly developed interferon- $\gamma($ IFN- $\gamma$ ) release assays (IGRA), e.g. the QuantiFERON ${ }_{\circledR}-\mathrm{TB}$ Gold test (QFN), are based on antigens found only in the $M$. tuberculosis complex and not in BCG strains [12-14] and use of these assays may yield more specific measures for ARTI estimation.

The apparent resurgence of clinical TB in Greenland prompted this study that aimed to determine the current prevalence of MTI and the underlying ARTI in school children and how much these estimates differ using either TST, QFN, or a combination of both. The study was performed in school children as M. tuberculosis infection in children reflects ongoing TB transmission. Additionally, the study aims to determine the dynamics of the current $M$. tuberculosis transmission by examining to what extent ARTI
AFFILIATIONS

*Dept of Epidemiology Research,

\# International Reference laboratory of Mycobacteriology, Statens Serum Institut,

${ }^{+}$Dept of Infectious Diseases, Rigshospitalet, University of Copenhagen, Copenhagen, Denmark, and

"Dept of Internal Medicine, Queen Ingrid's Hospital, Nuuk, Greenland.

CORRESPONDENCE

B. Soborg

Dept of Epidemiology Research

Statens Serum Institut

Artillerivej 5

DK- 2300 Copenhagen S

Denmark

E-mail: bot@ssi.dk

Received:

Jan 292010

Accepted after revision:

April 212010

First published online:

June 012010 
is influenced by sex, age, BCG vaccination programme and location.

\section{MATERIAL AND METHODS}

\section{Study site and population}

Greenland is arctic, and $82 \%$ of the country is covered by an ice cap, leaving only a narrow costal strip inhabited. The population comprises 57,000 people of which $90 \%$ are Inuit. Greenland is an integral part of the Kingdom of Denmark, but governed by home rule through a local government. Although less affluent than Danes in Denmark, the population is wealthy by the standards of developing countries and healthcare is operating at Danish standards [15].

The survey took place in five different towns and nearby settlements across Greenland: Tasiilaq (November, 2005) representing $56 \%$ of the population of Eastern Greenland; Narsaq (May, 2006), Qaqortoq (August, 2006) and Nanortalik (September, 2006) representing almost $100 \%$ of the population of Southern Greenland; and Sisimiut (May, 2007) representing $53 \%$ of the population of Western Greenland (excluding the capitol city of Nuuk). Each town was chosen on the basis of the annual TB incidence reported to the Chief Medical Officer in Greenland to represent different TB incidence localities; a high TB incidence area (South Greenland), middle TB incidence area (East Greenland) and low TB incidence area (West Greenland). For each town, data were collected over a 2-week period. All Greenlandic children follow mandatory schooling from age 6-16 yrs and all children within the school register were personally invited to participate in the study. 2 weeks in advance of each survey, the children received a personal letter of invitation, survey information, a consent sheet and a questionnaire to be filled in by the parents; all materials were both in Greenlandic and Danish. The surveys took place on predefined dates at the local schools where children were MTI examined along with their homeroom classmates. Whenever a child listed in the school protocol was not present on the day of the survey, the teacher was asked if the child was merely absent that day or did not attend the school anymore. Due to frequent migration of households in Greenland, a number of children listed in the school protocol no longer lived in the area. Whenever an absent child returned to school within the survey period, the child was asked by the teacher to join the survey. The study staff consisted of the physicians and authors B. Soborg, A.B. Andersen and K. Ladefoged, the TB research nurses, K. Laursen and K. Kristiansen and medical student, M. Veber.

Standardised, self-administered questionnaires completed by a parent or caregiver provided information on the family, living condition and health. Parental place of birth was used as an indicator of ethnicity. To be categorised as Inuit ethnicity, both of the parents should be registered as born in Greenland. The applied approach to ethnicity has previously been validated in Greenlandic studies from our group [16]. Information on place of birth was obtained from the Danish Civil Registration System (CRS) that contains demographic information on all inhabitants of Greenland as well as Denmark [17]. All information in the CRS is linked by a personal identification number given to all inhabitants at time of birth. Data from nonparticipating children in the survey towns were obtained from the CRS and the distribution of sex, age and ethnicity compared with the participating children.

\section{Testing for M. tuberculosis infection}

For detection of $M$. tuberculosis infection, the participants had venous blood samples drawn for the QFN and immediately after had a TST. The TST was applied to the dorsal aspect of the forearm by the intradermal Mantoux method using 2 tuberculin units $(0.1 \mathrm{~mL})$ of purified protein derivative (PPD; RT-23, SSI, Copenhagen, Denmark). $72 \mathrm{~h}$ after inoculation, the induration transverse to the long axis of the arm was read by an experienced examiner and the maximum diameter recorded in $\mathrm{mm}$. In accordance with local Greenlandic TB guidelines, an induration $\geqslant 12 \mathrm{~mm}$ was considered as a positive test result. The QFN was performed according to the manufacturer's instructions (Cellestis Ltd, Carnegie, Australia) [18] using Quantiferon software, version 2.33. High IFN- $\gamma$ background response occurred infrequently, with $3 \%$ of the samples presenting a nil value $\geqslant 0.35 \mathrm{IU} \cdot \mathrm{mL}^{-1}$ and only $1 \% \geqslant 1.0 \mathrm{IU} \cdot \mathrm{mL}^{-1}$. The amount of IFN- $\gamma$ produced in plasma was quantified in an ELISA. An IFN- $\gamma$ value $\geqslant 0.35 \mathrm{IU} \cdot \mathrm{mL}^{-1}$ obtained after TB antigen stimulation was considered positive and indicative of infection with $M$. tuberculosis. A test result was indeterminate if the IFN- $\gamma$ response, when corrected for background, was $<0.50 \mathrm{IU} \cdot \mathrm{mL}^{-1}$ in mitogen response and $<0.35 \mathrm{IU} \cdot \mathrm{mL}^{-1}$ in antigen response. A negative result was obtained by an IFN- $\gamma$ value $<0.35 \mathrm{IU} \cdot \mathrm{mL}^{-1}$.

\section{Definitions of MTI}

MTI was defined as a cutaneous induration of $\geqslant 12 \mathrm{~mm}$ in reaction to TST and an IFN- $\gamma$ response of $0.35 \mathrm{IU} \cdot \mathrm{mL}^{-1}$ or greater in the QFN, regardless of clinical status. Subjects with dual-negative TST and QFN results were defined as uninfected, i.e. MTI-negative. Subjects with discordant TST and QFN results were considered as probable MTI cases and not included in the main analyses.

\section{BCG vaccination status}

The official BCG vaccination programme in Greenland implied vaccination of all newborns born either before January 1, 1991, or after January 1, 1997. The BCG vaccination status of the participating children was obtained retrospectively through detailed vaccination protocols kept at the birthplace of the child. It was possible to obtain BCG vaccination records from $1,323(59.6 \%)$ children in the cohort, of whom $460(34.8 \%)$ had been BCG vaccinated.

\section{Ethical considerations}

The study fulfilled the Helsinki declaration and written informed consent was given by parents or guardians. All participants with either a positive TST or QFN result were referred to the local medical centre for further evaluation which included chest radiograph and general clinical evaluation to rule out ongoing TB. If clinical TB was diagnosed, the children were offered 6 months of standard TB treatment with isoniazid, rifampicin, pyrazinamide and ethambutol for 2 months, followed by isoniazid and rifampicin for 4 months. If active TB was excluded and the subject considered a case of MTI, a 6-month prophylactic isoniazid treatment was offered. The study was approved by the Commission for Scientific Research in Greenland, approval No. 505-105. 


\section{Statistical analysis}

The annual risk of MTI was estimated by modelling the binary status of MTI (infected, non-infected) in a non-linear binomial regression with the probability of infection modelled as $1-(1-\beta)^{\text {age }}$, where $\beta$ is the parameter representing the annual risk of MTI. The small number of children in the age groups $\leqslant 5$ and $>16$ yrs led us to restrict the estimation to the age groups 6-16 yrs. The annual risk estimation was based on the assumptions of no differential mortality between infected and non-infected and no effect of period. Estimation was performed using PROC NLMIXED in SAS (SAS institute, Cary, NC, USA). Strata-specific estimates were found by applying the model within strata and tests for homogeneity between strata were approximative F-tests. The cumulative risk of MTI at the18th birthday was estimated as $1-(1-\beta)^{18}$ with $95 \%$ CI determined by PROC NLMIXED. The association (expressed as risk ratios) between discordant QFN/TST results and demographic variables was analysed by log-linear binominal regression models. Agreement between QFN and TST was determined by Kappa statistics. The association between participation and demographic variables was analysed by logistic regression in the cohort of all citizens in the five areas. All p-values were two-sided, and $p<0.05$ was considered significant.

\section{RESULTS}

Of 2,880 school-registered children in the five survey towns, $2,218(77.0 \%)$ children were present at the time of study and completed a questionnaire. Demographic information on the participating children is shown in table 1. Participation rates by study area were as follows: Tasiilaq, 96\%; Narsaq, 79\%; Nanortalik, 89\%; Qaqortoq, 85\%; and Sisimiut, 57\%. The majority of the study participants $(1,958,88.3 \%)$ were Inuit, $1,117(50.4 \%)$ were male, and the mean \pm SD age among participants was $11.4 \pm 3.1$ yrs. In total, the sample population equalled $25 \%$ of the population of Greenland in the relevant age group (6-16 yrs) as registered in the CRS.

The ethnic distribution was equal among participating and non-participating children. The study population included fewer males $(49.9 \%$ among the participants as opposed to $53.9 \%$ among the non-participants; $\mathrm{p}=0.04$ ) and fewer older children, aged $\geqslant 14$ yrs (22.9\% among participants as opposed to $47.2 \%$ among non-participants; $\mathrm{p}<0.001$ ).

\section{Prevalence of MTI}

Of 2,218 included children 2,189 (98.7\%) had at least one of the two tests performed, and 1,886 (85.0\%) had both tests performed.

Blood samples were obtained from 2,117 (95.4\%) children and tested by QFN analysis, of which 197 (9.3\%) were positive and $22(1.0 \%)$ indeterminate. TST was successfully applied and read on 1,958 children $(88.3 \%)$, of which 205 (10.5\%) were considered positive. Among these children, the recorded induration was distributed as follows: $0 \mathrm{~mm}, 1,709(87.3 \%)$; 1-5 mm, 20 (1.0\%); 6-9 mm, $8(0.4 \%) ; 10 \mathrm{~mm}, 13(0.7 \%)$; $11 \mathrm{~mm}, 3$ (0.2\%); $12 \mathrm{~mm}, 6$ (0.3\%); $13 \mathrm{~mm}, 4$ (0.2\%); $14 \mathrm{~mm}, 2$ $(0.1 \%)$; and $\geqslant 15 \mathrm{~mm}, 193(9.9 \%)$. Among the 1,886 study participants tested with both tests, $152(8.1 \%)$ were concordant positive and considered M. tuberculosis infected. 250 (11.4\%)

\begin{tabular}{|c|c|c|}
\hline TABLE 1 & \multicolumn{2}{|c|}{$\begin{array}{l}\text { Demographic characteristics of } 2,218 \\
\text { Greenlandic school children screened for } \\
\text { Mycobacterium tuberculosis infection (MTI) }\end{array}$} \\
\hline All & & $2218(100.0)$ \\
\hline \multicolumn{3}{|l|}{ Sex } \\
\hline Male & & $1117(50.4)$ \\
\hline Female & & $1101(49.6)$ \\
\hline \multicolumn{3}{|c|}{ Age at examination yrs } \\
\hline $5-7$ & & $384(17.3)$ \\
\hline $8-10$ & & $603(27.2)$ \\
\hline $11-13$ & & 701 (31.6) \\
\hline $14-15$ & & $373(16.8)$ \\
\hline$\geqslant 16$ & & $157(7.1)$ \\
\hline \multicolumn{3}{|l|}{ Ethnicity } \\
\hline Inuit & & $1958(88.3)$ \\
\hline Caucasian & & $56(2.5)$ \\
\hline Mixed & & $204(9.2)$ \\
\hline \multicolumn{3}{|c|}{ Town and year of examination } \\
\hline Tasiilaq 200 & & $451(20.3)$ \\
\hline Narsaq 200 & & $285(12.9)$ \\
\hline Nanortalik 2 & & $410(18.5)$ \\
\hline Qaqortoq 2 & & $503(22.7)$ \\
\hline Sisimiut 200 & & $569(25.7)$ \\
\hline \multicolumn{3}{|c|}{ BCG vaccination status } \\
\hline Vaccinated & & $460(20.7)$ \\
\hline Non-vaccina & & $863(38.9)$ \\
\hline Missing ${ }^{\#}$ & & $895(40.4)$ \\
\hline \multicolumn{3}{|c|}{ QuantiFERON$N_{\circledast}$-TB Gold } \\
\hline Positive & & $197(8.9)$ \\
\hline Negative & & $1898(85.5)$ \\
\hline Indetermina & & $22(1.0)$ \\
\hline Missing & & $101(4.6)$ \\
\hline \multicolumn{3}{|c|}{ Tuberculin skin test } \\
\hline Positive & & 205 (9.3) \\
\hline Negative & & $1753(79.0)$ \\
\hline Missing ${ }^{+}$ & & $260(11.7)$ \\
\hline \multicolumn{3}{|l|}{ MTI } \\
\hline Positive $^{\S}$ & & $152(8.1)$ \\
\hline Negative & & $1645(87.3)$ \\
\hline Discordant & & $89(4.6)$ \\
\hline
\end{tabular}

Data are presented as n (\%). BCG: Bacille Calmette-Guérin; * : missing due to inability to obtain the participating child's BCG vaccination record. '? missing due to inability of obtaining a venous blood sample from the participating child. ${ }^{+}$: missing due to the inability of applying the tuberculin skin test or due to loss of follow up for test read-off. ${ }^{\text {s }}$ : positive test results estimated by concordant QuantiFERONB-TB Gold test and tuberculin skin test results.

children had either a positive QFN or TST. Figure 1 illustrates the M. tuberculosis infection prevalence by age groups.

\section{Annual risk of MTI}

Overall, the ARTI was estimated to be $0.80 \%$ (95\% CI $0.67-0.92 \%)$. In figure 1, the estimated $M$. tuberculosis infection prevalence based on a constant annual risk of $0.80 \%$ is displayed alongside the observed $M$. tuberculosis infection prevalence. The estimated 


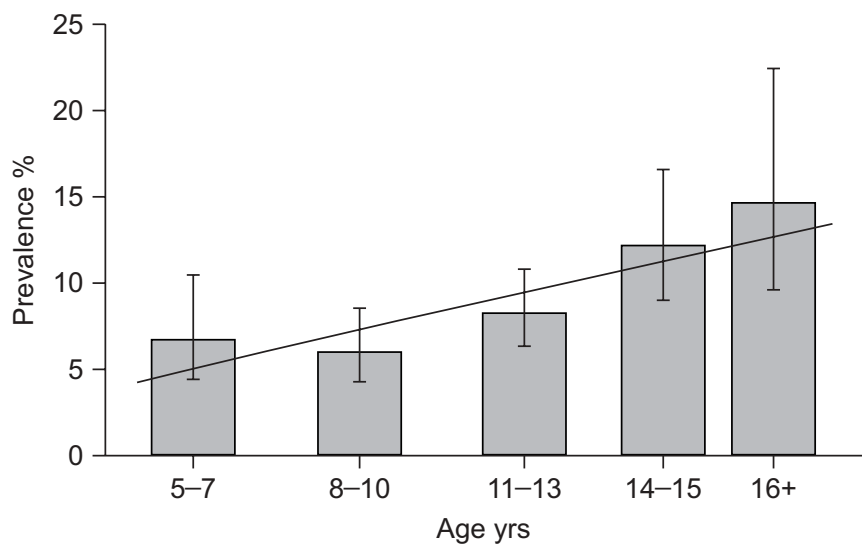

FIGURE 1. Observed and estimated Mycobacterium tuberculosis infection prevalence among 1,746 Greenlandic school children by age groups: Bars depict the observed $M$. tuberculosis infection prevalence by age group with confidence limits. The line marks the estimated $M$. tuberculosis infection prevalence calculated on the basis of a $0.80 \%$ annual risk of $M$. tuberculosis infection.

prevalence fits within the confidence limits of the observed prevalence of MTI for all age groups. The figure demonstrates that a constant ARTI, independent of age, fits well with the observed prevalence. On the basis of the $0.80 \%$ annual risk, the cumulative risk of MTI at the18th birthday equals $13.4 \%$ (95\% CI 11.4-15.4\%).

Table 2 shows the annual risk within strata of selected demographic variables. ARTI was independent of age, sex and BCG vaccination programme but associated with being of Inuit ethnicity $(0.87 \%$ (95\% CI $0.73-1.02 \%)$ ) in contrast to being of Caucasian or mixed inheritance $(0.02 \% \quad(95 \% \mathrm{CI}$ $0.00-0.37 \%))(p<0.001)$.
Figure 2 illustrates the ARTI in the five survey locations. A distinct inter-location variation was observed $(\mathrm{p}<0.001)$ with ARTI ranging from $0.13 \%(95 \%$ CI $0.03-0.24 \%)$, in the western town of Sisimiut, to $1.68 \%$ (95\% CI 1.26-2.10\%), in the southern town of Nanortalik.

The estimated annual risk of infection remained stable even when changing the TST cut-off points. Thus, at a TST cut-off point of $\geqslant 5 \mathrm{~mm}$, the ARTI was estimated to be $0.83 \%$ (95\% CI $0.70-0.97 \%$ ), at $\geqslant 6 \mathrm{~mm} 0.82 \%$ (95\% CI $0.69-0.95 \%)$, at $\geqslant 10 \mathrm{~mm}$ $0.80 \%(95 \%$ CI $0.67-0.93 \%)$, and at $\geqslant 15 \mathrm{~mm} 0.82 \%(95 \%$ CI $0.69-0.95 \%)$. The estimated annual risk increased slightly if based on the single-positive criterion derived from either TST or QFN. The ARTI was estimated to be $0.89 \%$ (95\% CI $0.76-$ $1.01 \%$ ) based on prevalence estimates using a single-positive QFN criterion and to be $0.99 \%$ (95\% CI $0.85-1.13 \%)$ using a single-positive TST criterion. However, the observed differences in the annual risk of MTI according to selected demographic variables as shown in table 2 and figure 2 using a dual-positive criterion were the same when using a singlepositive criterion.

\section{Concordance between the two MTI screening test modalities}

Concordance between TST and QFN is presented in table 3. 152 (8.1\%) had concordant positive TST and QFN results and 1,625 (86.2\%) children had concordant negative results. Thus, of 1,886 children tested with both tests, $94.3 \%$ had concordant test results yielding a Kappa agreement of 0.79 (95\% CI 0.74-0.84; p<0.001). Discordance was not associated with sex, but with older age (relative risk (RR) 1.17, 95\% CI 1.07-1.28) and prior BCG vaccination (RR 2.66, 95\% CI 1.53-4.68; p<0.0001). Kappa agreement for individuals with prior BCG vaccination was $0.68(95 \%$ CI $0.56-0.79 ; \mathrm{p}<0.001)$ and without prior vaccination $0.84(95 \%$ CI $0.78-0.91 ; \mathrm{p}<0.001)$.

TABLE 2 Estimates of annual risk of Mycobacterium tuberculosis infection among 1,746\# Greenlandic school children by sex age, ethnicity and Bacille Calmette-Guérin (BCG) vaccination status

\begin{tabular}{|c|c|c|c|c|}
\hline & $\begin{array}{c}\text { Children } \\
n\end{array}$ & $\begin{array}{c}\text { Dual test positive } \\
\text { n (\%) }\end{array}$ & $\begin{array}{c}\text { Annual risk } \\
(95 \% \mathrm{Cl})\end{array}$ & Test for homogeneity \\
\hline Sex & & & & $p=0.89$ \\
\hline Females & 883 & $73(8.3)$ & $0.79 \%(0.61-0.97)$ & \\
\hline Males & 863 & $73(8.5)$ & $0.80 \%(0.62-0.99)$ & \\
\hline$\geqslant 11$ yrs (i.e. $11-16 \mathrm{yrs}$ ) & 988 & $97(9.8)$ & $0.79 \%(0.63-0.94)$ & \\
\hline Ethnicity & & & & $p<0.001$ \\
\hline Inuit & 1545 & $142(9.2)$ & $0.87 \%(0.73-1.02)$ & \\
\hline Caucasian or mixed ethnicity ${ }^{\S}$ & 201 & $4(2.0)$ & $0.02 \%(0.00-0.37)$ & \\
\hline BCG vaccination $f$ & 1044 & & & $p=0.84$ \\
\hline Yes & 336 & $27(8.0)$ & $0.89 \%(0.55-1.22)$ & \\
\hline
\end{tabular}

\#: comprises children between 6-16 yrs of age with a dual tuberculin skin test/QuntiFERON $\mathrm{k}$-TB Gold test result. Children $<6$ and $>16$ yrs of age are excluded from the analyses due to scares number of children in the two groups. ": the age dichotomy is created based on the mean age of the participants which was 11.4 yrs. ${ }^{\S}$ : mixed ethnicity refers to children with both an Inuit and a Caucasian parent. ${ }^{f}$ : the total number of BCG vaccinated between $6-16$ yrs of age identified through protocol registration. 


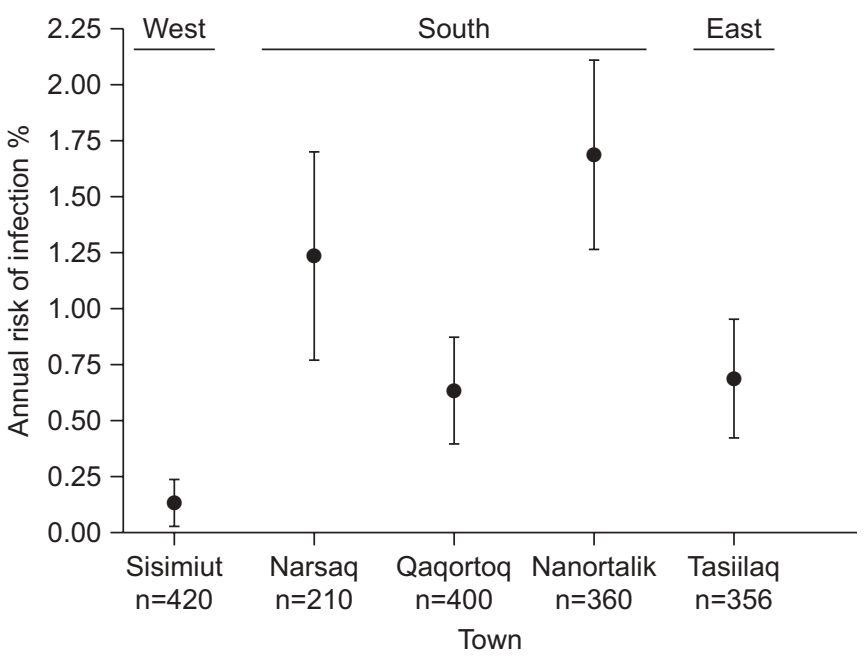

FIGURE 2. Annual risk of Mycobacterium tuberculosis infection (ARTI) among 1,746 Greenlandic school children by town. The ARTI of the five survey towns are depicted with $95 \% \mathrm{Cl}$ by geographic location (west, south and east).

22 children had an indeterminate QFN result and were analysed separately. The 22 indeterminate QFN results were all observed in the three youngest age groups (5-7, 8-10, 11-13 yrs), with 11 of the 22 indeterminate test results among the youngest children (5-7 yrs).

\section{Clinical TB cases}

Upon clinical follow-up of 250 test-positive children, four $(1.6 \%)$ Inuit children were diagnosed with clinical TB disease resulting in a TB prevalence of 4 out of 2,189 or 180 out of 100,000 (95\% CI 50-470). Two females, aged 8 and 16 yrs, were from the eastern town of Tasiilaq and two other females aged 8 and 16 yrs were from the southern town of Nanortalik. All four children had dual-positive test results.

\section{DISCUSSION}

The present investigation among children in Greenland documents a concerning high rate of $M$. tuberculosis transmission. The annual risk of transmission was $0.8 \%$ and by the 18 th birthday, $>13 \%$ of the children were estimated to have become infected. The problem was almost exclusively observed among
Inuits and clearly more severe in some geographical locales than others.

Although the extent of the problem among children was unknown until now, previous studies have reported TB incidence rates among Inuit populations from settlements in Greenland, Canada and Alaska that exceed the national rates of Denmark, Canada and the USA by 10-20-times [19-21]. The high rates of transmission among children document the fact that the efforts to control TB in recent years have been insufficient and $10 \%$ of the identified MTI children will, if left untreated, eventually progress to active disease and thereby maintain a high infectious burden. Already, four $(2.6 \%)$ of the 152 MTI children identified through this study had developed active tuberculous disease resulting in a TB prevalence as high as 180 out of 100,000 .

The annual risk was independent of age, sex and BCG vaccination programme, but pronounced ethnical and regional differences were observed. The substantial heterogeneity according to geographical area implies that conditions favouring spread within towns varied.

Our estimate of infection rates must be considered conservative, since we applied a restrictive criterion for infection. Thus, a person was only considered infected if found to be positive both in TST and QFN. The infection rate was higher if estimated using only the TST or the QFN when defining persons as infected. By using a dual-positive criterion for infection the ARTI estimate was shown to be independent of the TST cut-off point. The ARTI estimate was slightly higher if based only on TST than when based on QFN. However, both estimates were independent of sex, age and BCG vaccination programme, and both higher for Inuit. Thus, both tests were found to be adequate tools for following rate changes. However, it is to be noted that $24 \%$ of the TST-positive subjects were QFN-negative or indeterminate. If some of the discordant results comprised by TST-positive and QFNnegative subjects were due to BCG vaccination, as suggested by the associations we found, then the QFN implies better specificity of infection. Because a positive result will lead to six-month isoniazid treatment of an otherwise healthy child, the improved specificity and possibly sensitivity of the QFN may be of clinical value. Furthermore the QFN exhibited logistic advantages compared with TST, as TST required the

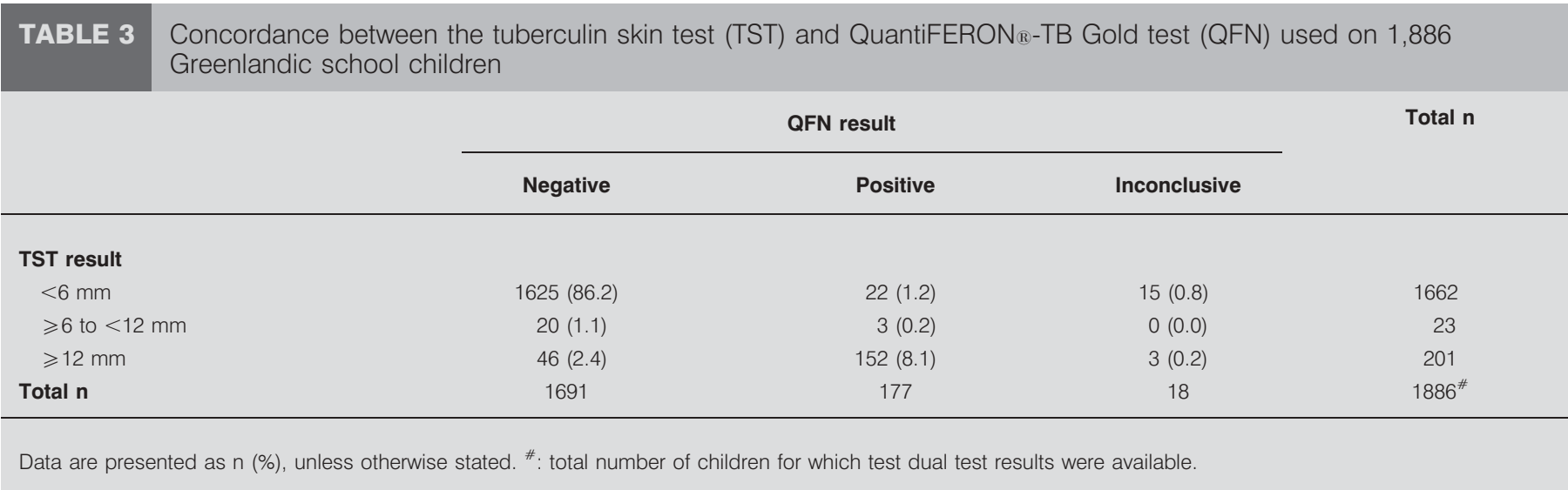


participants to return for assessment after $72 \mathrm{~h}$, and some participants failed to return.

In addition to a stringent criterion for infection, the study had several other strengths. It was based on a large sample of children comprising $\sim 25 \%$ of the entire Greenlandic population in the relevant age groups. The study had a high participation rate and was designed to represent children within the different geographical locales in Greenland. Information regarding participants was partly retrieved in mandatory national registries minimising information bias. However, certain limitations should be considered. The number of BCG vaccinated children could be underestimated due to lack of protocol registration. Furthermore, there could be a bias in the study population due to non-participation of sick children not attending school. This would tend to underestimate the true burden of disease caused by infection with $M$. tuberculosis. Also there could be an underestimation of the risk of infection due to prior prophylactic treatment given during earlier TB screening surveys as prior treatment can cause children to revert to a negative TST or a negative QFN [22].

Information from questionnaires may be subjected to recall bias, although, as TB infection status was unknown to the participants, we believe the misclassification to be nondifferential; thus, if anything, underestimating the true effect. The endemic TB "flares" around the world, such as is present in Greenland illustrate the multifaceted problems underlying TB control. In the developing world, the increase in TB incidence is associated with HIV/AIDS [23, 24], and is further complicated by multidrug-resistant $M$. tuberculosis strains [25] and, recently, extensively drug-resistant strains [26, 27]. Compared with the developing world, Greenland is a relatively wealthy country with a healthcare system equal to Scandinavian countries [15]. The healthcare system functions well, providing equal care to all citizens free of charge. In Greenland, the TB resurgence is not attributable to HIV/AIDS or multidrug-resistant strains as, so far, no multidrug resistance and only a few HIV-related TB cases have been reported [4]. However, the results from this study show that TB is being transmitted at an alarmingly high rate and that the country faces a serious challenge in the years to come if this situation is to be reverted. The reasons for the high TB transmission and continuing high TB incidence are largely unexplained; however, perhaps the environmental factor of a harsh polar climate, which forces indoor activity for a large proportion of total time, could be part of the explanation. Tubercle bacilli expelled indoors have a long survival and hence, can potentially be the cause of a greater number of infections than bacilli expelled outdoors [28]. Furthermore, the traditional Inuit way of family life favours large families and, with a shortage of housing in Greenland, night-time crowding in poorly maintained houses becomes inevitable. The lifestyle conditions within Greenland could explain the more "difficultto-control" tuberculosis situation observed in the country, even with the same standard of healthcare compared with continental Denmark. Even so, the survey highlights the challenges of TB control programmes to further reduce the $\mathrm{TB}$ burden, once the initial phase of epidemic control has surpassed $[1,2]$.
In conclusion, the present study documented a very high annual risk of transmission of $M$. tuberculosis among Inuit children in Greenland and the finding emphasizes the urgent need for a strengthening of the National tuberculosis programme. Prompt interventions are warranted and targeted preventive efforts aimed at the Inuit population within the identified high burden localities could be beneficial. The broader lesson learned from the Greenlandic example is the importance of maintaining a well-functioning TB surveillance programme for decades after an apparent decline in incidence, especially in prior high incidence areas, such as the circumpolar region.

\section{SUPPORT STATEMENT}

The study was funded by The Commission for Scientific Research in Greenland (Nuuk, Greenland), the Danish Research Council, the Greenlandic Home Rule Government, Cluster in International Health, Faculty of Health Sciences, University of Copenhagen, the Danish Lung Association, the Thorvald Madsen Foundation, Fonden til Lægevidenskabens fremme, the Ebba Celinder Foundation. The sponsor played no role in the study design; the collection, analysis or interpretation of data; the writing of the report; or in the decision to submit the manuscript for publication.

\section{STATEMENT OF INTEREST}

None declared.

\section{ACKNOWLEDGEMENTS}

We would like to thank R. Biggar (Statens Serum Institut, Copenhagen, Denmark) for his valuable comments on an earlier version of this paper. Furthermore, we thank TB-Research nurses K. Laursen (Queen Ingrids Hospital, Nuuk, Greenland), K. Kristiansen (Queen Ingrids Hospital) and J. Weismann (Tasiilaq District Hospital, Tasiilaq, Greenland) and medical student M. Veber (University of Copenhagen, Copenhagen) for help in conducting the screening surveys across Greenland. We would like to thank laboratory technician J.H. Pedersen and her colleagues at the International Reference laboratory of Mycobacteriology (Statens Serum Institut, Copenhagen, Denmark) for processing the QFN samples. We also thank T. Skifte at the Chief Medical Office, Nuuk, for generating TB notification data. Finally, we thank the staff at the schools and health care facilities for their co-operation.

\section{REFERENCES}

1 Grzybowski S, Styblo K, Dorken E. Tuberculosis in Eskimos. Tubercle 1976; 57: S1-S58.

2 Stein KS. Tuberculosis in Greenland and the Fight Against It. Copenhagen, Danish National Union Against Tuberculosis and Lung Disease, 1994; pp. 7-56.

3 Soborg C, Soborg B, Pouelsen S, et al. Doubling of the tuberculosis incidence in Greenland over an 8-year period (1990-1997). Int J Tuberc Lung Dis 2001; 5: 257-265.

4 Chief Medical Officer in Greenland. Annual report from the Chief Medical Officer in Greenland. Nuuk, Chief Medical Officer in Greenland, 2007.

5 Rieder H. Annual risk of infection with Mycobacterium tuberculosis. Eur Respir J 2005; 25: 181-185.

6 Dye C, Watt CJ, Bleed DM, et al. Evolution of tuberculosis control and prospects for reducing tuberculosis incidence, prevalence, and deaths globally. JAMA 2005; 293: 2767-2775.

7 Cauthen GM, Pio A, ten Dam HG. Annual risk of tuberculous infection. 1988. Bull World Health Organ 2002; 80: 503-511.

8 Kritzinger FE, den Boon S, Verver S, et al. No decrease in annual risk of tuberculosis infection in endemic area in Cape Town, South Africa. Trop Med Int Health 2009; 14: 136-142. 
9 Arnadottir T, Rieder HL, Trebucq A, et al. Guidelines for conducting tuberculin skin test surveys in high prevalence countries. Tuber Lung Dis 1996; 77: 1-19.

10 Huebner RE, Schein MF, Bass JB Jr. The tuberculin skin test. Clin Infect Dis 1993; 17: 968-975.

11 Farhat M, Greenaway C, Pai M, et al. False-positive tuberculin skin tests: what is the absolute effect of BCG and non-tuberculous mycobacteria? Int J Tuberc Lung Dis 2006; 10: 1192-1204.

12 Andersen P, Munk ME, Pollock JM, et al. Specific immune-based diagnosis of tuberculosis. Lancet 2000; 356: 1099-1104.

13 Rothel JS, Andersen P. Diagnosis of latent Mycobacterium tuberculosis infection: is the demise of the Mantoux test imminent? Expert Rev Anti Infect Ther 2005; 3: 981-993.

14 Pai M, Zwerling A, Menzies D. Systematic review: T-cell-based assays for the diagnosis of latent tuberculosis infection: an update. Ann Intern Med 2008; 149: 177-184.

15 Young K., Bjerregaard P., eds. Health Transitions in Arctic Populations. Toronto, University of Toronto Press, 2008; pp. 1-485.

16 Krause TG, Koch A, Poulsen LK, et al. Atopic sensitization among children in an arctic environment. Clin Exp Allergy 2002; 32: 367-372.

17 Pedersen CB, Gotzsche H, Moller JO, et al. The Danish Civil Registration System: a cohort of eight million persons. Dan Med Bull 2006; 53: 441-449.

18 Cellestis. www.cellestis.com. Date last updated: 2009. Date last accessed: October 2009.

19 Smeja C, Brassard P. Tuberculosis infection in an Aboriginal (First Nations) population of Canada. Int J Tuberc Lung Dis 2000; 4: 925-930.
20 Gaudette LA, Ellis E. Tuberculosis in Canada: a focal disease requiring distinct control strategies for different risk groups. Tuber Lung Dis 1993; 74: 244-253.

21 Funk EA. Tuberculosis control among Alaska Native people. Int J Tuberc Lung Dis 1998; 2: S26-S31.

22 Chee CB, Khinmar KW, Gan SH, et al. Effect of TB treatment on Tcell interferon- $\gamma$ responses to $M$. tuberculosis-specific antigens. Eur Respir J 2010; 36: 355-361.

23 Odhiambo JA, Borgdorff MW, Kiambih FM, et al. Tuberculosis and the HIV epidemic: Increasing annual risk of tuberculous infection in Kenya, 1986-1996. Am J Public Health 1999; 89: 1078-1082.

24 Corbett EL, Watt CJ, Walker N, et al. The growing burden of tuberculosis: global trends and interactions with the HIV epidemic. Arch Intern Med 2003; 163: 1009-1021.

25 Dye C, Espinal MA, Watt CJ, et al. Worldwide incidence of multidrug-resistant tuberculosis. J Infect Dis 2002; 185: 1197-1202.

26 Basu S, Andrews JR, Poolman EM, et al. Prevention of nosocomial transmission of extensively drug-resistant tuberculosis in rural South African district hospitals: an epidemiological modelling study. Lancet 2007; 370: 1500-1507.

27 Gandhi NR, Moll A, Sturm AW, et al. Extensively drug-resistant tuberculosis as a cause of death in patients co-infected with tuberculosis and HIV in a rural area of South Africa. Lancet 2006; 368: $1575-1580$.

28 Rieder HL. Epidemiology of tuberculosis in Europe. Eur Respir J 1995; 8: Suppl. 20, 620s-632s. 